\title{
Release of Acetylcholine from Embryonic Neurons Upon Contact with Muscle Cell ${ }^{1}$
}

\author{
I. CHOW ${ }^{2}$ AND M-M. POO
}

Department of Physiology and Biophysics, University of California, Irvine, California 92717

\begin{abstract}
When a spherical muscle cell (myoball) was manipulated into contact with either the soma or the neurite of an isolated neuron in 2-day-old Xenopus nerve-muscle cultures, depolarizations similar to miniature endplate potentials (MEPPs) were frequently detected in the muscle cell. These depolarizations occurred within minutes after myoball-soma contact and within seconds after myoball-neurite contact. They had time course and amplitude distribution similar to those of the MEPPs recorded from naturally occurring neuromuscular synapses between neurites and muscle cells found in the same cultures, but they occurred at a lower frequency and had smaller average amplitudes. These depolarizations were induced by acetyicholine (ACh) since they were reversibly blocked by addition of $d$-tubocurarine into the culture, and they were abolished in muscle cells pretreated with $\alpha$-bungarotoxin before contact with the neuron. Greater than $60 \%$ of the neuronal population in these cultures released $\mathrm{ACh}$ upon this direct muscle contact. The appearance of MEPP. like potentials in the myoball upon contact with an isolated neuron suggests that the cellular machinery responsible for $A C h$ release is present throughout the neuron and that packages of ACh molecules are available for release prior to nerve-muscle synapse formation. We also found that neurons which had previously made synapse with other muscle cells in the culture all failed to release ACh from the soma and showed reduced release capability at the neurite for the first $30 \mathrm{~min}$ to $1 \mathrm{hr}$ of contact with a myoball. This finding suggests that, during synapatogenesis, there is a depletion of $\mathrm{ACh}$ molecules and/or substances responsible for the triggering of their release in the extrasynaptic regions of the neuron.
\end{abstract}

In embryonic nerve-muscle culture, synapse formation is a rapid process: spontaneous miniature endplate potentials (MEPPs) and evoked endplate potentials (EPPs) can be recorded from a muscle cell within a few minutes of contact between the growth cone of an appropriate neuron and the skeletal muscle cell (Kidokoro and Yeh, 1982). In order to understand the initial events that lead to the establishment of synaptic transmission, it is advantageous to have a system in which the timing and the extent of nerve-muscle contact

Received June 11, 1984; Revised September 28, 1984;

Accepted October 3, 1984

${ }^{1}$ We thank Dr. Steven $\mathrm{H}$. Young for helpful discussion and comments on the manuscript. This work was supported by National Institutes of Health Grant NS-17558-02 and National Science Foundation Grant BNS 83-12348.

${ }^{2}$ To whom correspondence should be sent, at her present address: Jerry Lewis Neuromuscular Research Center, UCLA School of Medicine, Los Angeles, CA 90024. can be subjected to experimental manipulation. In the present study we manipulated isolated spherical embryonic Xenopus muscle cells (myoballs) into contact with various regions of co-cultured neurons while monitoring continuously the electrical activity of the muscle cells. Depolarizations similar to MEPPs were frequently observed in the myoballs within minutes after the contact was made with the soma, and within seconds when contact was made along the neurite or with the growth cone of an isolated neuron. These depolarizations appear to represent an early form of acetylcholine (ACh) release prior to the establishment of a functional synapse. We here describe the properties of this ACh release from isolated neurons as well as from neurons that had previously contacted other muscle cells. Preliminary results of this work have been reported in short form (Chow and Poo, 1983; Chow, 1984).

\section{Materials and Methods}

Cell culture and manipulation. Cells from the neural tube and myotomes of Xenopus embryos at stages 17 to 19 (Nieuwkoop and Faber, 1967) were dissociated and plated on clean glass microscope slides following a procedure previously described (Poo et al., 1978). In these cultures muscle cells of two distinct morphologies were observed: flat, spindle-shaped and spherical-shaped. The spherical muscle cells (myoballs) appeared as a result of the absence of a strongly adhesive substratum on the glass. Both spindleand spherical-shaped cells have a similar resting membrane potential, ACh sensitivity, and membrane input resistance (Orida and Poo, 1978; Chow and Poo, 1984). They show a similar capability to establish electrotonic coupling with other muscle cells (Chow and Poo, 1984), and both are capable of establishing functional synapses with co-cultured neurons (M-m. Poo, unpublished observation). Neurons were identified by the presence of neurites, and both isolated and muscle-contacted neurons were used. All cultures were maintained and all experiments were carried out on 2-day-old cultures at room temperature $\left(21\right.$ to $\left.23^{\circ} \mathrm{C}\right)$.

Two-day-old cultures were viewed with phase contrast optics of an inverted microscope (Nikon), and manipulation of the myoballs was done with glass microelectrodes controlled by a micromanipulator (Leitz). An isolated myoball was impaled with the microelectrode, detached from the glass surface by gently moving the micropipette in various directions, and then translocated into contact with the soma or the neurite of an isolated or a muscle-contacted neuron. The contacting cell surfaces between the soma and the myoball had a flat profile, and caution was taken so that the muscle cell would not touch any part of any extending neurite of the neuron. Neuritemyoball contact was assured when slight pressure applied on the muscle cell moved the neurites (usually the neurites are not fully attached onto the glass surface along their entire length). Contact between the growth cone and the myoball was made by placing the myoball on top of the growth cone and applying slight pressure on the myoball (see Fig. 1).

Electrophysiological methods. Standard intracellular recordings were carried out using Steinberg's solution $(58.2 \mathrm{mM} \mathrm{NaCl}, 0.7 \mathrm{mM} \mathrm{KCl}, 0.4 \mathrm{mM}$ $\mathrm{Ca}\left(\mathrm{NO}_{3}\right)_{2} \cdot \mathrm{H}_{2} \mathrm{O}, 1.3 \mathrm{~mm} \mathrm{MgSO} 4 \cdot 2 \mathrm{H}_{2} \mathrm{O}, 4.6 \mathrm{~mm}$ Tris, $250 \mathrm{IU}$ of penicillinstreptomycin, $\mathrm{pH} 7.8$ ), supplemented with $10 \mathrm{~mm} \mathrm{CaCl}_{2}$ for stabilization of microelectrode penetration (Spitzer, 1976). The intracellular recording pipette was filled with $3 \mathrm{M}$ potassium acetate and had resistances in the range of 70 to 110 megohms. The myoball was kept in contact with the neuron for periods up to $1 \mathrm{hr}$, and membrane potential changes were displayed on a storage oscilloscope (Tektronix) and continuously recorded with an FM taperecorder (Tandberg) or a strip chart recorder (Gould-2200). MEPP-like activity 

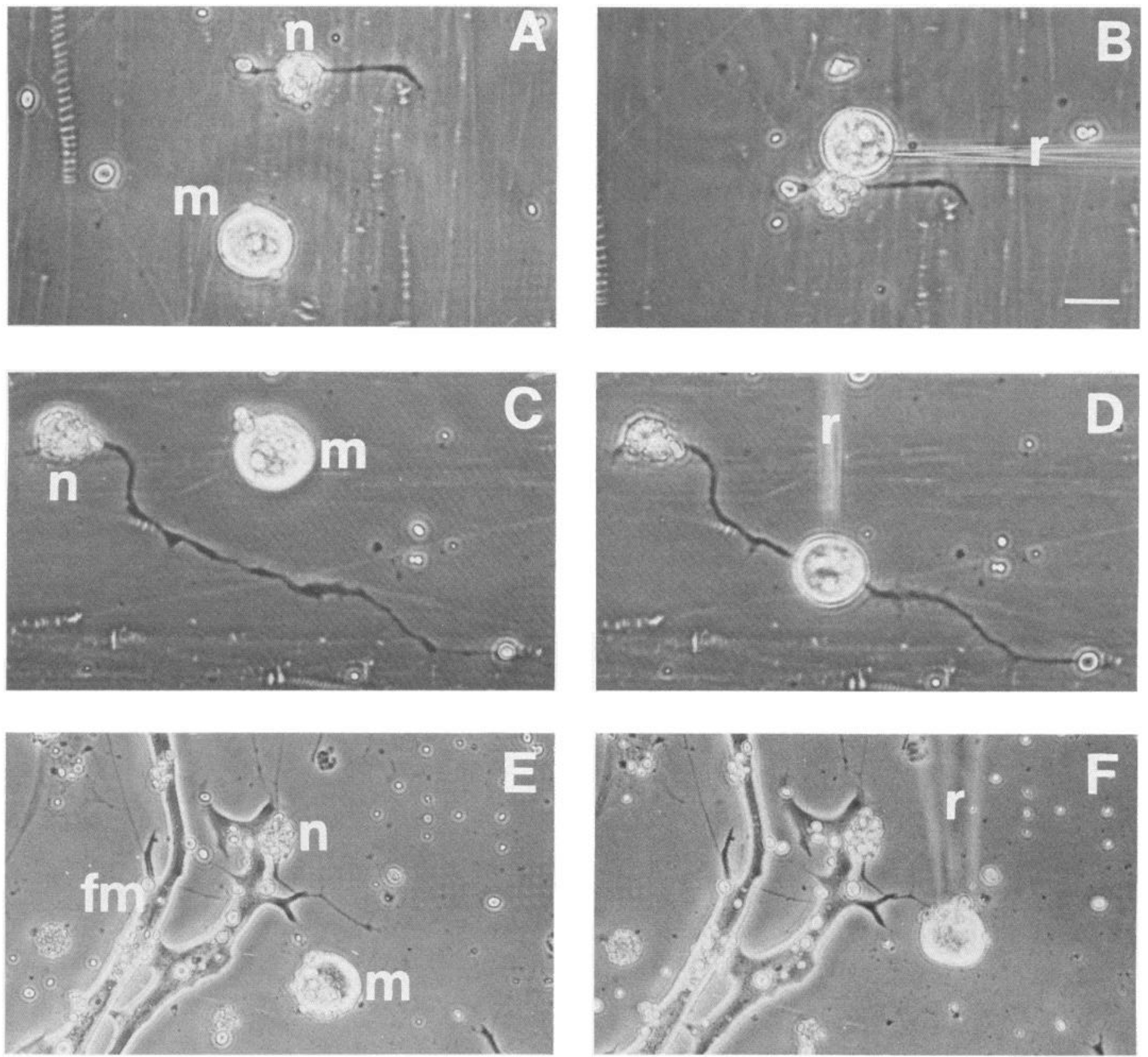

Figure 1. Phase contrast photographs of nerve and muscle cells taken from 2-day-old cultures. $A$, An isolated bipolar neuron and an isolated myoball before contact. $B$, The myoball was impaled with an intracellular microelectrode, detached from the glass culture substratum, and manipulated into contact with the soma of a neuron. $C$ and $D$, Operation similar to that in $A$ and $B$, but the contact of the myoball was made at the neurite. $E$, An isolated myoball and a multipolar neuron which had made functional contact with flat muscle cells $(\mathrm{fm})$. $F$, The myoball was impaled and then manipulated into contact with the growth cone of the neuron. Scale $=20 \mu \mathrm{m}$. $m$, muscle cell; $n$, neuron; $r$, intracellular microelectrode. 

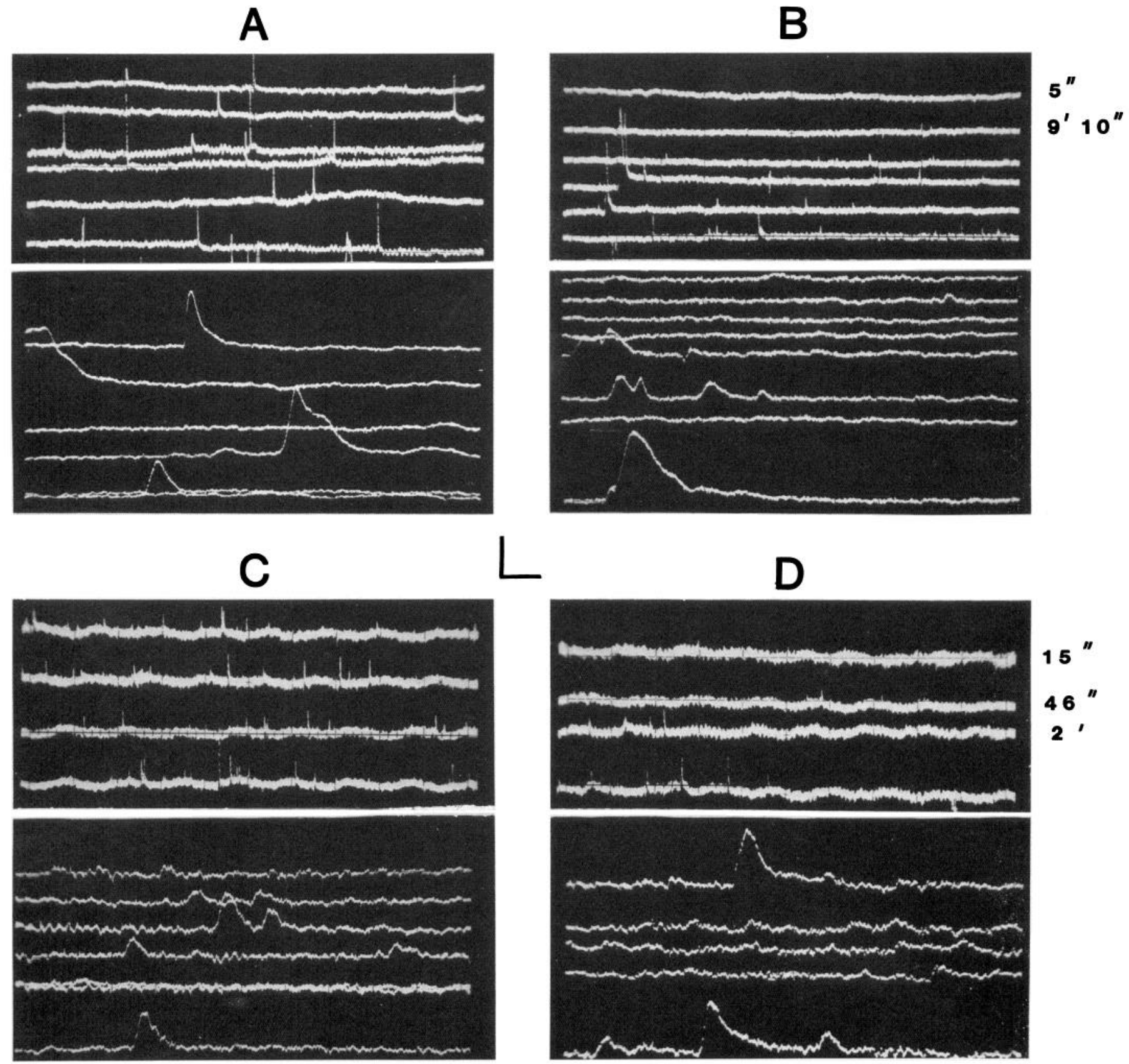

\section{$46^{\prime \prime}$

Figure 2. Photographs of oscilloscope tracings of myoball membrane potentials. The oscilloscope tracings are playback from tape recordings taken from a naturally occurring synapse $(A)$, an induced soma-myoball contact $(B)$, an induced neurite-myoball contact of an isolated neuron $(C)$, and an induced neurite-myoball contact of a muscle-contacted neuron $(D)$. Myoball membrane potentials at various times after the onset of myoball-neuron contact are shown in the upper block of tracings of each set (sweep speed: $0.5 \mathrm{sec} / \mathrm{div}$, voltage amplification: $10 \mathrm{mV} / \mathrm{div}$ ). The lower block of tracings of each set shows representative MEPP-like depolarizations at a faster sweep speed $(20 \mathrm{msec} / \mathrm{div})$ and a higher voltage amplification (4 mV/div). In C, all of the tracings are recordings made immediately after contact. In $B$ and $D$, the numbers mark the times (after the onset of contact) at which the corresponding recordings were made. Unmarked tracings in $B$ and $D$ are recordings immediately after the preceding marked tracings. Notice that in $B$, the first MEPP-like depolarization was detected at $9 \mathrm{~min}, 10 \mathrm{sec}$ after the onset of the contact. In $C$, depolarizations were detected immediately upon contact. In $D$, they appeared after 15 $\mathrm{sec}$ of contact. 
TABLE I

Properties of MEPP-like potentials in Xenopus neuron-muscle cultures

Intracellular recording of muscle membrane potentials were performed on five groups of Xenopus neuron-muscle pairs: (1) spontaneous neurite-muscle contacts found in the cultures; neuron-muscle contacts produced by manipulating a myoball into contact with an isolated (2) soma or (3) neurite; and manipulated contact betwen a myoball and (4) the soma or (5) the neurite of a neuron that has already established functional synapse with another muscle cell.

\begin{tabular}{|c|c|c|c|c|c|c|}
\hline & $\begin{array}{l}\text { Total No. } \\
\text { of Cell Pairs } \\
\text { Examined }\end{array}$ & $\begin{array}{c}\text { No. of Pairs } \\
\text { with MEPP-like } \\
\text { Potentials }\end{array}$ & $\begin{array}{l}\text { No. of Pairs } \\
\text { without MEPP-like } \\
\text { Potentials }\end{array}$ & $\begin{array}{c}\text { Average } \\
\text { Amplitude }^{a}\end{array}$ & $\begin{array}{l}\text { Average } \\
\text { Frequency }^{a}\end{array}$ & Time to Peak ${ }^{a}$ \\
\hline & & \multicolumn{2}{|c|}{$\%$ of total } & $\mathrm{mV}$ & events/sec & msec \\
\hline 2. Manipulated soma-myoball contacts & 95 & $\begin{array}{c}58 \\
(61 \%)\end{array}$ & $\begin{array}{l}37^{b} \\
(39 \%)\end{array}$ & $3.5 \pm 1.3$ & $0.09 \pm 0.02$ & $5.3 \pm 0.5$ \\
\hline 3. Manipulated neurite-myoball contacts & 33 & $\begin{array}{c}23 \\
(70 \%)\end{array}$ & $\begin{array}{c}10^{c} \\
(30 \%)\end{array}$ & $2.2 \pm 0.5$ & $0.27 \pm 0.12$ & $4.0 \pm 0.4$ \\
\hline 4. Manipulated soma-myoball contacts & 26 & $\begin{array}{c}0 \\
(0 \%)\end{array}$ & $\begin{array}{c}26^{b} \\
(100 \%)\end{array}$ & & & \\
\hline 5. Manipulated ncurite-myoball contacts & 36 & $\begin{array}{c}21 \\
(58 \%)\end{array}$ & $\begin{array}{c}15^{c} \\
(42 \%)\end{array}$ & $1.2 \pm 0.1$ & $0.32 \pm 0.11$ & $4.3 \pm 0.6$ \\
\hline
\end{tabular}

\footnotetext{
${ }^{a}$ Average values were obtained from random samples of 6 to 14 cell pairs (see the text). All values are \pm SEM.

${ }^{b}$ Contact period $\geq 20 \mathrm{~min}$.

${ }^{\circ}$ Contact period $\geq 3 \mathrm{~min}$
}

was defined as a depolarization larger than the background variation (range 0.2 to $0.7 \mathrm{mV}$ ) and showed fast risc of depolarization followed by a slowcr repolarization. If no such depolarizations occurred in the muscle cell for a period of 15 to $30 \mathrm{~min}$, the recording was discontinued. If depolarizations were observed, recording was carried out for a period up to $1 \mathrm{hr}$. Data were discarded when the muscle cell started to swell and/or when the membrane potential changed by more than $10 \mathrm{mV}$. Intracellular recordings were also carried out at established, naturally occurring neuromuscular synapses in these cultures using identical methods.

\section{Results}

Soma-myoball contact. In the first set of experiments, isolated myoballs were manipulated into contact with the soma of isolated neurite-bearing neurons in the culture (Fig. 1, $A$ and $B$ ). Figure $2 B$ depicts the myoball membrane potential during a typical experiment: no depolarization was seen immediately after contact, with the first depolarizations appearing at $9 \mathrm{~min}, 10 \mathrm{sec}$ after contact. High speed oscilloscope tracings (Fig. $2 B$, lower traces) show that these depolarizations resemble the MEPPs observed at established neuromuscular contacts in these same cultures. Examples of the latter MEPPs at a naturally occurring synapse are shown in Figure $2 A$.

Of the 95 soma-myoball cell pairs tested, 58 pairs $(61 \%)$ showed first appearance of small depolarizations within 20 min of contact (Table I), and of those 58 pairs, $33(57 \%)$ did so in less than $5 \mathrm{~min}$ of contact. Figure $3 A$ is the time-to-onset histogram of these depolarizations at soma-myoball contacts. No apparent correlation was found between the neuronal morphology (i.e., whether it was monopolar, bipolar, or multipolar) and the ability to induce MEPP-like depolarizations in the muscle cells. These Xenopus cultures contain a heterogeneous population of neurons, and the fraction of $\mathrm{ACh}$ releasing neurons, approximately $60 \%$, is close to the previously suggested values for cholinergic neurons in this type of culture (Cohen et al., 1979; Cohen and Weldon, 1980; Young and Poo, 1983).

Neurite-myoball contact. In the second set of experiments, impaled myoballs were pushed against neurites of isolated neurons (Fig. 1, $C$ and $D$ ), while the muscle cell membrane potential was monitored continuously. In contrast to the results from soma-muscle contact, MEPP-like activities appeared in many cells with a much shorter delay, within seconds after the contact was made. Figure $2 \mathrm{C}$ shows an example of recording obtained from these experiments. In this particular case depolarizations were detected immediately upon contact. The frequency of depolarizations in neurite-myoball pairs was higher than that usually found at soma-myoball contacts
(Table I). Of 33 neurite-myoball contacts tested, 21 showed response within $4 \mathrm{~min}$. The time-to-onset histogram is shown in Figure 3B. No obvious differences were found for contacts made at the different regions along the neurite or at the growth cone. Furthermore, in several cases, including contacts between the growth cone and the myoball, the delay time was smaller than the time resolution of the present study ( $1 \mathrm{sec}$; see inset of Fig. $3 B$ ).

Properties of MEPP-like potentials. The MEPP-like depolarizations observed after neuron-myoball contact were due to release of $\mathrm{ACh}$ from the neuron. This was suggested by results from two other experiments. First, in eight separate trials, $d$-tubocurarine was added to the cultures (at $30 \mu \mathrm{g} / \mathrm{ml}$ ) atter these depolarizations had been observed. In each case, all of the depolarizations were abolished within a few minutes. In three cases, when curare was washed out with fresh medium, recovery of depolarizations was observed. Second, the cultures were incubated for $30 \mathrm{~min}$ with saline containing $10 \mu \mathrm{g} / \mathrm{ml}$ of $\alpha$-bungarotoxin, a toxin known to block nicotinic ACh receptors. Subsequent contacts between myoball and soma (six pairs) and between myoball and neurite (four pairs) of identified cholinergic neurons all failed to induce MEPP-like depolarizations in these muscle cells. Furthermore, addition of tetrodotoxin $(30 \mu \mathrm{M})$, which is known to block $\mathrm{Na}^{+}$-dependent action potentials, did not abolish these depolarizations. These results are consistent with the notion that the observed depolarizations were due to spontaneous release of packages of $\mathrm{ACh}$ molecules from the neuron.

These miniature ACh potentials (MAPs) possess many properties similar to those of the MEPPs previously observed at neurite-muscle synapses in this culture (Kidokoro et al., 1980; Kidokoro and Yeh, 1982). For direct comparison, we have also examined the properties of MEPPs of naturally occurring neurite-muscle synapses in the same or parallel cultures. As shown in Table I, the average peak amplitude of the MAPs was $3.5 \pm 1.3 \mathrm{mV}$ ( \pm SEM, 11 cell pairs) for soma-myoball contacts, and $2.2 \pm 0.5 \mathrm{mV}$ ( \pm SEM, 9 cell pairs) for neurite-myoball contacts, values smaller than the MEPPs recorded from the same cultures, $7.6 \pm 1.0 \mathrm{mV}$ ( \pm SEM, 6 cell pairs). The average time to peak of the MAPs was similar to that of the MEPPs ( 4 to $5 \mathrm{msec}$ ). Figure 4 depicts the amplitude distribution of MEPPs and MAPs, recorded from a pre-existing synapse (Fig. $4, A$ and $B$ ), a soma-myoball contact (Fig. 4, $C$ and $D$ ), and a neurite-myoball contact (Fig. 4E). In most of the cases, the amplitude distribution of these MEPPs and MAPs had a skewed profile (Fig. 4, $A, D$, and $E$ ), showing a predominance of smaller amplitudes. Widespread, non- 

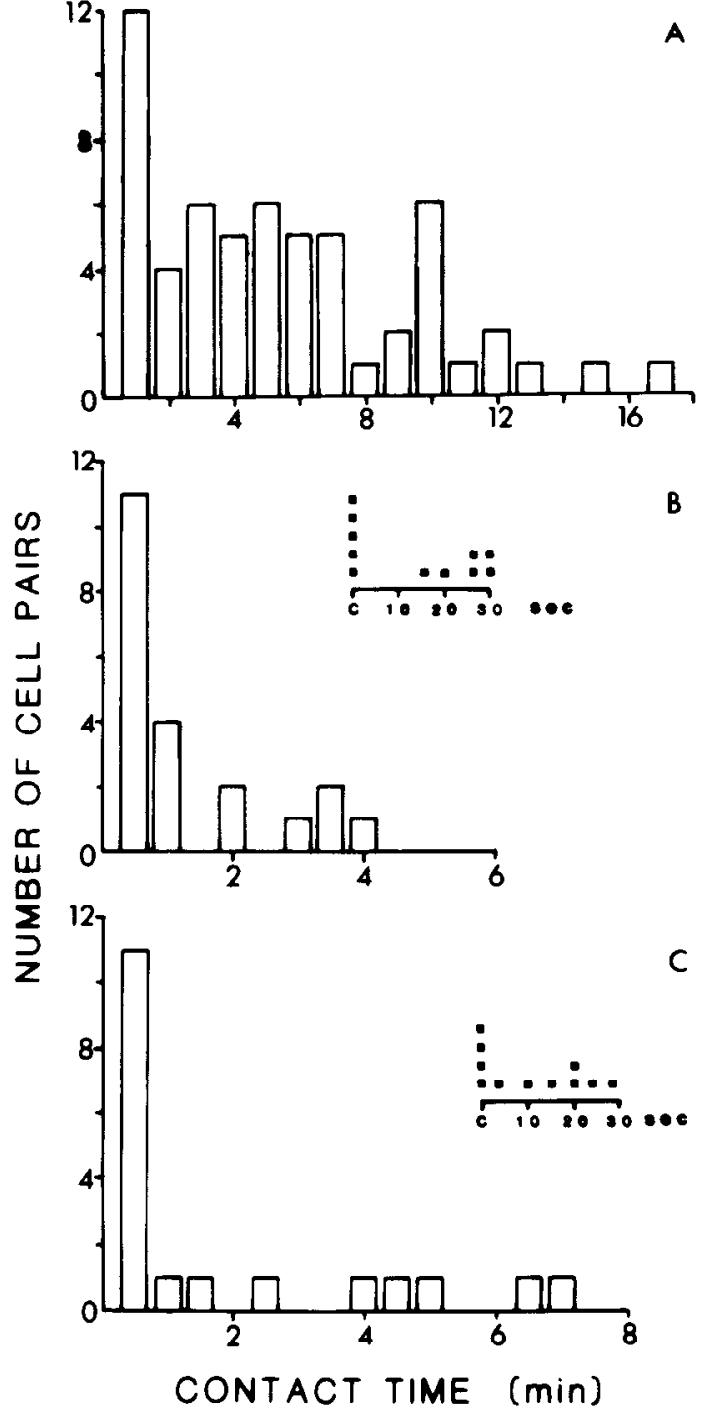

Figure 3. Histograms of the lapse time between the beginning of contact and the time for the first appearance of MEPP-like depolarizations at somamyoball contacts $(A)$ and neurite-myoball contacts of isolated neurons $(B)$ and neurite-myoball contacts of neurons which had previously formed functional synapse with other muscle cells $(C)$. Insets in $B$ and $C$ show the distribution of lapse time within the first $30 \mathrm{sec}$ of contact.

skewed profile was occasionally seen for both MEPPs and MAPs (Fig. 4, B and C).

The average frequency of occurrence of MAPs was much lower than that of MEPPs (Table I): $0.09 \pm 0.02$ events/sec ( \pm SEM, 14 pairs) for soma-myoball pairs (range, 0.02 to 0.28 events/sec) and $0.27 \pm 0.12$ events/sec ( \pm SEM 11 pairs) for neurite-myoball contacts (range, 0.02 to 1.4 events/sec), as compared to $0.53 \pm 0.17$ events/sec ( \pm SEM, 6 pairs) for spontaneously formed synapses (range, 0.22 to 1.37 events/sec). Figure 5 shows changes of MAP frequency with time after contact for several soma-myoball (Fig. 5A) and neurite-myoball (Fig. $5 B$ ) cell pairs. No apparent pattern in the change of MAP frequency was observed: the frequency remained basically unaltered for some pairs, whereas it varied a great deal for other pairs.

Refractoriness for ACh release after synaptogenesis. Many neurons in these co-cultures make contact and establish functional synapse with both spindle and spherical muscle cells, as shown by the presence of the MEPPs, EPPS, and, occasionally, muscle contractions. Not all naturally occurring nerve-muscle contacts form functional synapses: about $30 \%$ of the tested neurite-contacted muscle cells showed no MEPPs during prolonged stable recording up to $30 \mathrm{~min}$ (Table I). In these cases, the neurons are likely to be noncholinergic.

When neurons which had previously formed functional synapses with other muscle cells were tested for ACh release upon contact with a myoball, the results were clearly different from those described above for the isolated neurons. In these experiments, functional innervation was first assessed by the appearance of spontaneous twitching and/or MEPPs in the neurite-contacted muscle cells. The new soma-myoball or neurite-myoball contact was then produced (Fig. 1F) and MAPs were recorded in an identical manner as described above. The results are shown in the last two rows of Table I. Interestingly, for all 26 new soma-myoball contacts no MAP activity was detected for contact periods of 20 to $50 \mathrm{~min}$. As for the new neurite-myoball contacts, the fraction of neurons that showed release was only $58 \%$ ( 21 of 36 pairs). Notice that all these 36 neurons tested were cholinergic, since they all had functional synaptic transmission with the muscle cells already innervated by them. Figure $2 D$ depicts a sample of oscilloscope tracings for such a neurite-myoball contact. The average MAP amplitude was half of that found at contacts with isolated neurons; but both the average frequency and time to peak were similar to the values found at contacts between muscle and neurites of isolated neurons (Table I). Similar to the isolated neurons, those muscle-contacted neurons which responded to myoball contact did so rapidly (Fig. 3C), each pair with its own frequency characteristics (Fig. 5C), and the MAP amplitudes also had a skewed distribution (Fig. 4F). Apparently, the prior functional contact with a muscle cell alters the properties of the neuron in such a way that it becomes less capable of releasing $\mathrm{ACh}$ at a new contact site.

\section{Discussion}

In this study, we have demonstrated that both the soma and the neurite of isolated Xenopus embryonic neurons are capable of releasing packages of $\mathrm{ACh}$ molecules, and that the release can be detected by a muscle cell in contact with the neuron. Since it has been shown that isolated embryonic neurons-at least the growth cones - are capable of releasing ACh prior to their contact with the muscle cell (Hume et al., 1983; Young and Poo, 1983), our observed MEPP-like depolarizations at the contacting myoballs could simply be reflecting the spontaneous ACh-releasing process. In addition, the manipulated contact with a muscle cell may have increased this spontaneous release

The occurrence of MEPP-like depolarizations in the muscle cells does not necessarily imply the establishment of functional synaptic transmission. In preliminary experiments, we stimulated the soma electrically with an extracellular patch electrode after the MEPP-like depolarizations had appeared and monitored the muscle membrane response. In eight neuron-myoball cell pairs (three for soma-myoball and five for neurite-myoball) tested, only one pair showed EPP-like response in the myoball. This situation appears to be similar to that which occurs during the early stages of endplate reinnervation. Dennis and Miledi (1974) reported the existence of "non-transmitting" synapses at the regenerating neuromuscular junctions of frog cutaneous pectoris muscle that exhibited spontaneous MEPPs without nerve-evoked EPPs. In a similar Xenopus culture, Kidokoro and Yeh (1982) found that, a few minutes after the growth cone had contacted the muscle cell, synaptic potentials were evoked by stimulation of the neurite, but spontaneous MEPPs were rarely observed in the muscle. The reason for the difference between the findings of the latter study and those reported here is probably the fact that Kidokoro and Yeh (1982) were only monitoring contacts between the growth cone and the muscle cell. Furthermore, it is possible that the pressure exerted by the myoball on the neurons in the present study may have also increased the spontaneous release of $\mathrm{ACh}$, and such a pressure is absent in a naturally occurring growth conemuscle contact

Release of neurotransmitter from postganglionic cell bodies has 


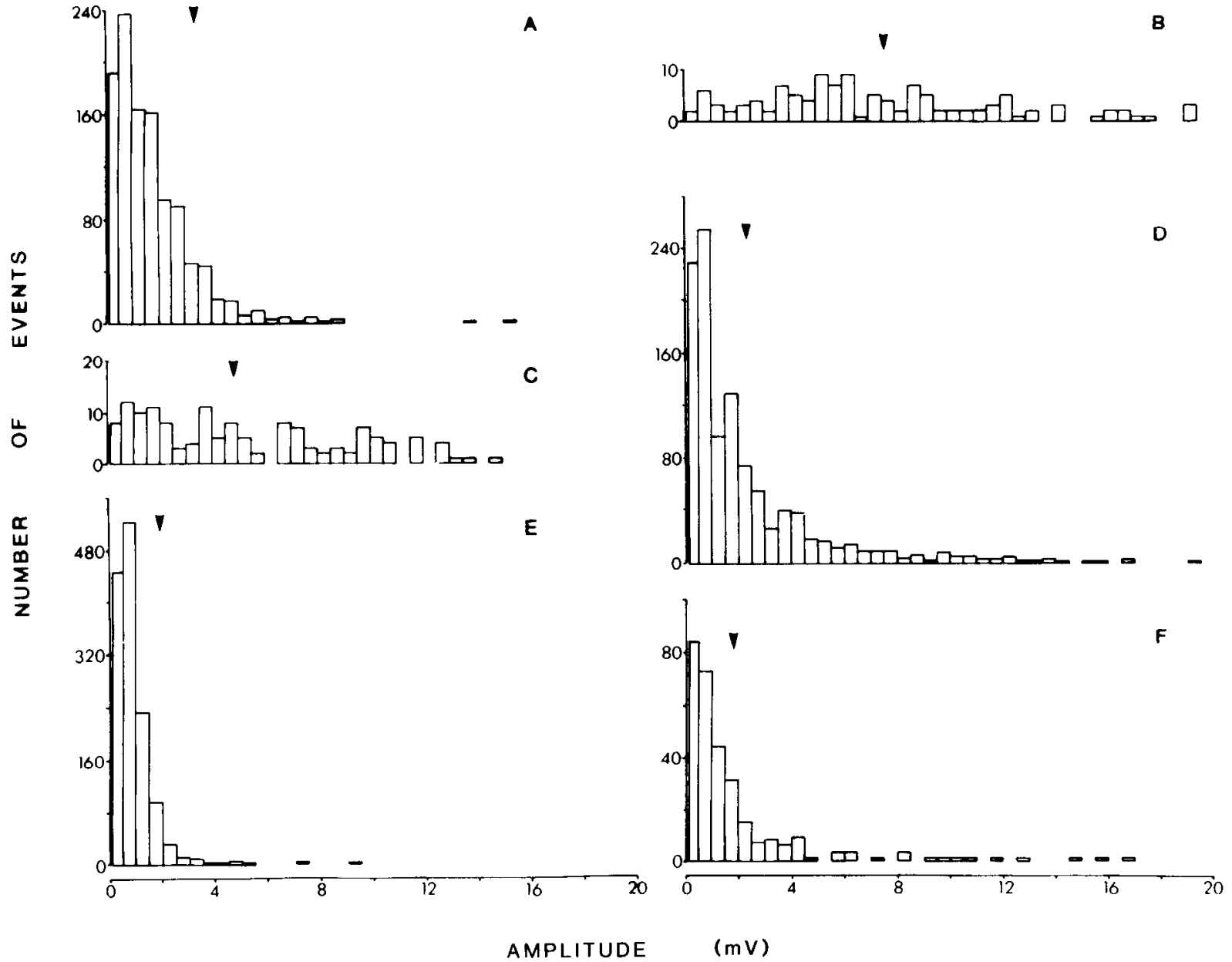

Figure 4. Histograms of the amplitude distribution of MEPPs at naturally occurring, functional nerve-muscle synapses $(A$ and $B)$, and MEPP-like depolarizations at soma-myoball contacts $(C$ and $D)$ and neurite-myoball contact of an isolated neuron $(E)$, and at the neurite-myoball contact of a musclecontacted neuron $(F)$. Data for each histogram were recorded from one myoball, and the arrowhead points to the average amplitude for all of the MEPPs or MEPP-like events. The total number of events counted for each histogram was between 119 and 1099 .

been described in isolated frog sympathetic ganglia after postganglionic antidromic stimulation (Koketsu et al., 1972; Suetake et al., 1981), and in denervated chick ciliary ganglion in response to $\mathrm{K}^{+}$ depolarization, antidromic stimulation, and cholinergic agonists (Johnson and Pilar, 1980). These previous results and the present finding of $A C h$ release from the soma all suggest the presence of a somatic transmitter pool which can be released directly via the somatic membrane. Since the soma normally does not serve as a presynaptic element, the function of somatic transmitter release is not apparent. During the early phase of neurite outgrowth from an embryonic neuron, the soma is likely to be the site for the synthesis and packaging of $\mathrm{ACh}$ molecules, and the membrane components responsible for controlling the transmitter release are also likely to be widespread in the entire membrane, as suggested by the high lateral mobility of membrane components in the embryonic neurons (Levi et al., 1980; Patel and Poo, 1982). The capability of transmitter release throughout the entire neuron may reflect a general plasticity of the growing neuron for the future site of synaptic contact on its surface. This notion is further supported by our finding that prior contact with another muscle cell rendered refractoriness to the neuronal soma and decreased $A C h$ release at the neurites. The loss of release capability could reflect either a redistribution of $A C h$ synthesis and packaging machinery within the neuronal cytoplasm and/or a differentiation of neuronal plasma membrane. Neuronal contact on the Xenopus muscle cell is known to induce a redistribution of muscle cell surface ACh receptors during the first day of nerve-muscle co-cultures, resulting in an accumulation of the ACh receptors at the site of neurite-muscle contact (Anderson et al., 1977). It is possible that a similar redistribution of membrane proteins also occurs in the neuronal membrane as a process of presynaptic differentiation. A contact-site localization of neuronal membrane proteins responsible for the recognition of the muscle cell or for $\mathrm{ACh}$ release could account for the apparent refractoriness observed in the present study.

Although these results suggest that the observed ACh release occurs in packages, as shown by the MEPP-like discrete depolarizations, we do not know whether it reflects the exocytosis of synaptic vesicle-like structures. Vesicles resembling synaptic vesicles have been found along the neurites and at some growth cones of these cultured neurons (Weldon and Cohen, 1979), but whether they contain ACh molecules and whether they are involved in the induction of these MEPP-like depolarizations is not known. The observed amplitude histograms showed distinct skewed distribution toward the lower amplitudes, which is characteristic of the MEPPs observed at developing synapses (Kullberg et al., 1977; Kidokoro et al., 1980; Kidokoro, 1984) and denervated endplates (Birks et al., 1960; Miledi, 1960). Our results are consistent with the notion that the observed depolarizations result from the exocytosis of partially filled precursors of ACh-containing synaptic vesicles. Alternatively, they could also be due to spontaneous opening of neuronal membrane "channels" which allow the pulsatile release of cytoplasmic ACh molecules in 

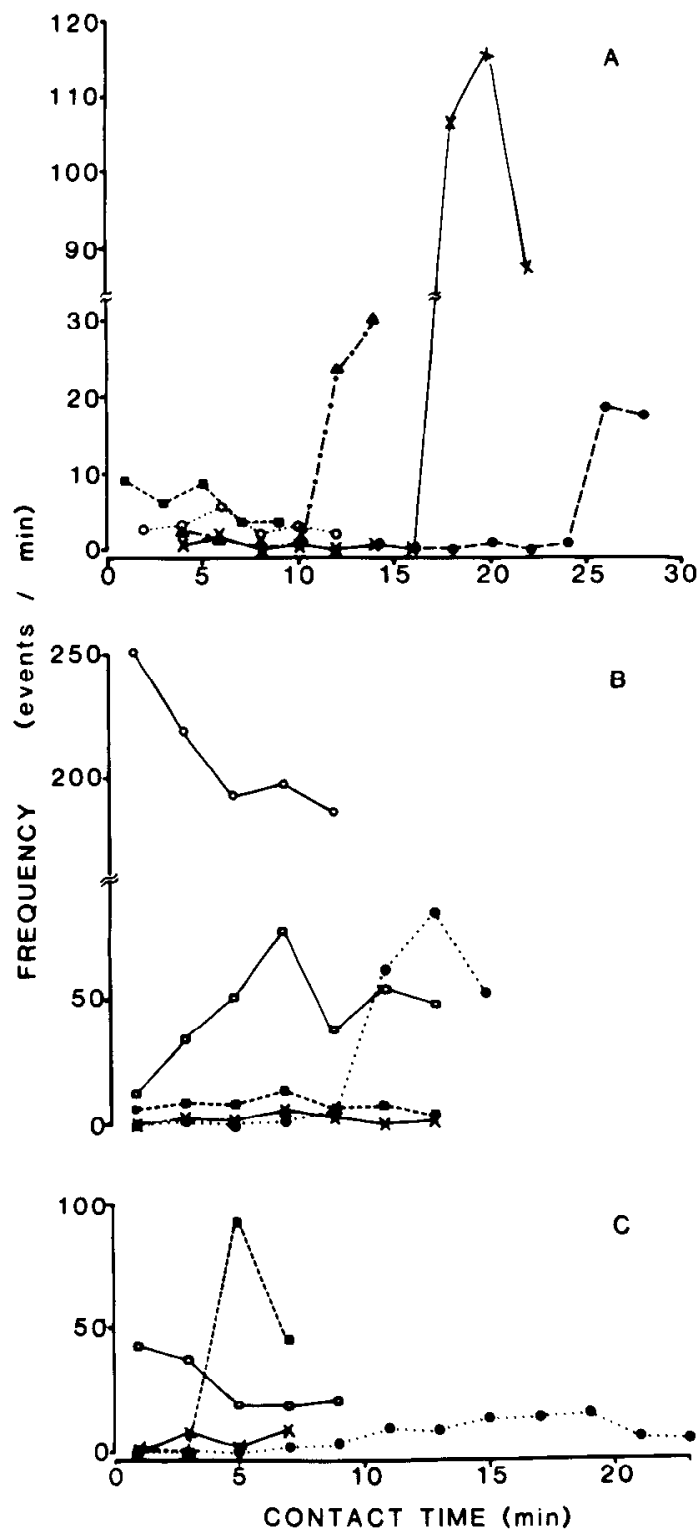

Figure 5. Temporal changes in the frequency of MEPP-like potentials after contact at soma-myoball contacts $(A)$ and neurite-myoball contacts of isolated neurons $(B)$, and neurite-myoball contacts of neurons that had previously formed functional synapse with other muscle cells $(C)$. Different symbols represent data obtained from different cell pairs.

the neuron (Johnson and Pilar, 1980; also see discussion by Tauc, 1982).

\section{References}

Anderson, M. J., M. W. Cohen, and E. Zorychta (1977) Effects of innervation on the distribution of acetylcholine receptors on cultured muscle cells. $\mathrm{J}$. Physiol. (Lond.) 268: 731-756.

Birks, R., B. Katz, and R. Miledi (1960) Physiological and structural changes at the amphibian myoneural junction in the course of nerve degeneration. J. Physiol. (Lond.) 150: 145-168.
Chow, I. (1984) Apparent neuronal refractoriness to muscle contact after synaptogenesis. Soc. Neurosci. Abstr. 10: 582.

Chow, I., and M-m. Poo (1983) Rapid induction of acetylcholine release from the neuronal soma by muscle contact. Soc. Neurosci. Abstr. 9: 688.

Chow, I., and M-m. Poo (1984) Formation of electrical coupling between embryonic Xenopus muscle cells in culture. J. Physiol. (Lond.) 346: 181194.

Cohen, M. W., and P. R. Weldon (1980) Localization of acetylcholine receptors and synaptic ultrastructure at nerve-muscle contacts in culture: Dependence on nerve type. J. Cell Biol. 86: 388-401.

Cohen, M. W., M. J. Anderson, E. Zorychta, and P. R. Weldon (1979) Accumulation of acetylcholine receptors at nerve-muscle contacts in culture. Prog. Brain Res. 49: 335-349.

Dennis, M. J., and R. Miledi (1974) Non-transmitting neuromuscular junctions during an early stage of endplate reinnervation. J. Physiol. (Lond.) 239: 553-570.

Hume, R. I., L. W. Role, and G. D. Fischbach (1983) Acetylcholine release from growth cones detected with patches of acetylcholine receptor-rich membranes. Nature 305: 632-634.

Johnson, D. A., and G. Pilar (1980) The release of acetylcholine from postganglionic cell bodies in response to depolarization. J. Physiol. (Lond.) 299: 605-619.

Kidokoro, Y. (1984) Two types of miniature endplate potentials in Xenopus nerve-muscle cultures. Neurosci. Res. 1: 157-170

Kidokoro, Y., and E. Yeh (1982) Initial synaptic transmission at the growth cone in Xenopus nerve-muscle cultures. Proc. Natl. Acad. Sci. U. S. A. 79: 6727-6731.

Kidokoro, Y., M. J. Anderson, and R. Gruener (1980) Changes in synaptic potential properties during acetylcholine receptor accumulation and neurospecific interactions in Xenopus nerve-muscle cell culture. Dev. Biol. 78: 464-483.

Koketsu, K., S. Minota, and M. Nakamura (1972) The presynaptic potential produced by postsynaptic activation in bullfrog sympathetic ganglion. Life Sci. 11: 263-268

Kullberg, R. W., T. L. Lentz, and M. W. Cohen (1977) Development of the myotomal neuromuscular junction in Xenopus laevis: An electrophysiological and fine-structural study. Dev. Biol. 60: 101-129.

Levi, A., Y. Shechter, E. J. Neufeld, and J. Schlessinger (1980) Mobility, clustering, and transport of nerve growth factor in embryonal sensory cells and in a sympathetic neuronal cell line. Proc. Natl. Acad. Sci. U. S. A. 77 3469-3473.

Miledi, R. (1960) The acetylcholine sensitivity of frog muscle fibres after complete or partial denervation. J. Physiol. (Lond.) 151: 1-23.

Nieuwkoop, P. D., and J. Faber (1967) Normal Table of Xenopus laevis (Daudin), Elsevier-North Holland Publishing Co., Amsterdam.

Orida, N., and M-m. Poo (1978) Electrophoretic movement and localisation of acetylcholine receptors in the muscle cell membrane. Nature 275: 3135.

Patel, N., and M-m. Poo (1982) Orientation of neurite growth by extracellular electric fields. J. Neurosci. 2: 483-496.

Poo, M-m. W-j. H. Poo, and J. W. Lam (1978) Lateral electrophoresis and diffusion of concanavalin $A$ receptors in the membrane of embryonic muscle cell. J. Cell Biol. 76: 483-501.

Spitzer, N. (1976) The ionic basis of the resting potential and a slow depolarizing response in Rohon-Beard neurons of Xenopus tadpoles. J. Physiol. (Lond.) 255: 105-135.

Suetake, K., H. Kojima, K. Inanaga, and K. Koketsu (1981) Catecholamine is released from non-synaptic cell-soma membrane: Histochemical evidence in bullfrog sympathetic ganglion cells. Brain Res. 205: 436-440.

Tauc, L. (1982) Nonvesicular release of neurotransmitter. Physiol. Rev. 62 857-893.

Weldon, P. R., and M. W. Cohen (1979) Development of synaptic ultrastructure at neuromuscular contacts in an amphibian cell culture system. $\mathrm{J}$. Neurocytol. 8: 239-259.

Young, S. H., and M-m. Poo (1983) Spontaneous release of transmitter from growth cones of embryonic neurones. Nature 305: 634-637. 